\title{
Pushing music: People's continued will to archive versus Spotify's will to make them explore
}

\author{
Author: \\ Marika Lüders, Department of Media and Communication, University of Oslo, Norway \\ Contact information: \\ Marika Lüders, Department of Media and Communication, P.O.Box 1093 Blindern, \\ 0317 Oslo. E-mail: marika.luders@media.uio.no
}

\begin{abstract}
Music streaming services provide people with access to vast libraries of music, but also encourage certain patterns of consuming music. In this article I use Spotify as a case and investigate the action potentials for exploring and archiving music. The personal role of music implies we may expect the 'will to archive' to be prevalent even if these archives are not based on individual ownership. First, an analysis of Spotify suggests the machine agency of Spotify pushes people towards exploring music, whereas archiving features are material and depend of human action. Spotify is hence skewed towards prompting users to explore rather than archive music. Next, an analysis of 23 focus-group interviews suggests that users value opportunities to explore music, yet their practices are equally directed towards archiving music. Theoretically, this article delineates how objects with machine agency are different from material objects in terms of affordances. The action potentials of material objects are symmetrically constituted by what the objects provide relative to an active being. The action potentials of objects with machine agency interfere with this symmetry: the machine is designed to act on behalf of the human being, making certain affordances more perceivable than others.
\end{abstract}

Keywords: Music streaming; Spotify; affordance; materiality; machine agency; human practice; archive

\section{Introduction}

With more than 30 million songs available in most music streaming services, how do people choose what music to listen to? Do they explore the vast library of available music or stick with familiar tunes? Such questions go beyond the individual user to also include the economics and politics of streaming services, where certain user-patterns might be more desirable for the stakeholders delivering the service. This article uses Spotify as a case and explicates action potentials for saving and exploring music. I argue that Spotify is designed to push users towards exploring music whereas users still remain attached to their personal music history. Granted, Spotify enables people to explore music and to maintain music collections. The database of available music, 
recommendation algorithms, and social features for discovering music through peers provide users with opportunities to explore music. Features for saving albums and artists and for creating playlists give people means to maintain collections of 'their music'. Yet I propose that Spotify is designed to make actions for exploring music more perceivable. Unlike features for saving music, features for exploring move beyond being mere features and become autonomous actors in people's music consumption. This skew towards exploring comes with a cost: streaming music leaves no permanent trace unless the listener takes further actions.

Music collections are firmly tied to personal meanings and life narratives (DeNora, 2000; Giles et al., 2007; Kibby, 2009; van der Hoeven, 2018). Music experiences often co-occur with a certain time and place, and may later acquire an affective power as musical indices, evoking memories of the past while at the same time taking on new layers of meaning (Turino, 1999). Yet musical memories are subject to the trajectories of life (taking on additional meanings), and are intricately interwoven between personal and collective memory (van Dijck, 2006). We may hence expect users of music streaming services to remain attached to their musical preferences and history; organizing music into repositories, even if these are not physical or based on individual ownership, and even if these repositories are at the risk of disappearance if the service closes down.

Whereas the database of Spotify is an ordered and flexible archive, the 'database available in the cloud breaks the dialectic of subjectivity and objectivity, providing a system of ordering that is produced by someone else' (Marshall, 2014: 68). We thus need to separate between the archive of the service provider and the individual's need for her/his own archive. As Featherstone proposes (2006: 595), 'the will to archive is a powerful impulse in contemporary culture', and the volume of digital information makes the will to archive ever more pressing. With a reference to Simmel, Featherstone argues that we have everything, but possess nothing, and we yearn for a framework through which we can make sense of the volume and speed of cultural products (Featherstone, 2000: 162-163). Whereas 'archive' in terms of a subscription-based access (and not ownership) to music may appear as an ill-fitted notion, we can still expect the 'will to archive' to be present among Spotify-users.

I have outlined an argument where the need and will to retain music in streaming services is explained as a way to make sense of the vast volume of content, and as a way to safeguard the personal meaning of music. This line of reasoning rests upon what Hesmondhalgh (2008) identifies and criticises as the dominant conception of music, emotion and identity, where music is conceived as a 'positive resource for active self-making' (Hesmondhalgh, 2008: 330). While people may indeed mobilise music in positive ways, Hesmondhalgh points to how an adequate conception of music and identity needs to enquire how such goals of self-realisation are linked with power. The self as defined and constituted partly by the (cultural) products we consume, align well with neo-liberalist conceptions of the self as an individual enterprise. Hesmondhalgh acknowledges that music matters for people, yet he reminds us to examine musical 
consumption and how music matters, or come to matter, as imbued with the values of self-realisation.

The objective of this paper is to investigate exploring and archiving music from the point of view of Spotify as a service provider on the one hand and people on the other. This implies paying attention to what happens in the space between the technology and the user. For this purpose, the notion of affordance is a pertinent analytical lens. Gibson's (1979) theory of affordances has recurrently been proposed to enable a third way between technological determinism and social constructionism (Hutchby, 2001; Leonardi, 2011; Nagy and Neff, 2015; Schrock, 2015) by acknowledging the technical capacities of an object as well as human perceptions of how that object could be used. An analysis of affordances requires investigating music streaming services both as matter and as human practice. I hence raise the following research questions:

RQ1: How does Spotify facilitate exploring and archiving music?

RQ2: How do people express needs for exploring and archiving music?

I address RQ1 by extracting key-points from the extant literature in an analysis of Spotify as a music streaming service. This discussion is followed by an analysis of qualitative data from Spotify-users on their needs and experiences with exploring and archiving music (RQ2).

\section{Theoretical framework and related research}

Gibson (1979) defines affordance as what an object offers an animal or a human being. Affordances are not the object's physical properties, and they must always be considered relative to the acting being. A seat or a bench looks sit-on-able if this object is perceived as horizontal, flat, rigid and knee-high relative to a perceiver. Hence, the sit-on-able affordance of a bench is neither an objective nor a subjective property: 'It is equally a fact of the environment and a fact of behaviour' (Gibson, 1979: 179). Gibson argues that what we perceive when we look at objects are primarily their affordances. For example, when we look at a bench, we perceive it as sit-on-able, more than its separate physical qualities. The question in terms of Spotify is consequently, what do we perceive when we look at this service? As affordances are constituted in relationships between people and the materiality of the technology, this question cannot be addressed by examining human practice alone, or by examining materiality alone. It is an elusive concept; affordances relate to matter or the objective features and functions, yet affordances and objective features are not the same.

Affordances point to the action-potentials of an object, yet in order to examine and understand affordances, it is essential to start with the matter. My emphasis on matter as crucial for understanding affordance is an important positioning statement when compared to how affordance is typically appropriated in communication studies. Dreading the label 'technological determinists', communication scholars neglect the character of affordances as always relational, always both object and perceiver; and end 
up with an appropriation of the term that refers to how humans choose to use technology (Nagy and Neff, 2015). Given that 'an affordance is a relationship between the properties of an object and the capabilities of the agent that determine just how the object could possibly be used' (Norman, 2013: 11), I delineate the properties of the software (materiality) and how humans perceive and act upon these properties.

\section{What's the matter with Spotify?}

Enquiring how technological artefacts matter and how technical capacities constrain and enable certain actions represent a contrast to the constructivist emphasis on how technologies are ultimately socially constructed (Hutchby, 2000). Materiality extends beyond physical artefacts to include the materiality of software in terms of the constraints and actions they enable (Hutchby, 2010; Leonardi, 2010). The legacy of this line of reasoning can be traced to Actor-Network-Theory (ANT) and the core objective to 'reassemble the social' to include non-human entities (Latour, 2005). We need to understand non-human entities as network-nodes that affect and have consequences for the social and for the society (Latour, 2005; Law, 1992).

Exploring the materiality of a given type of software means delineating what features are pertinent for different tasks (Leonardi, 2010). The subsequent question is what constitutes a pertinent level of analysis when identifying and discussing the materiality of software. To what extent do we need to uncover the technical details, the architecture with clients, storage systems or backend sites? Or does it suffice to pinpoint the functionality and features as presented through the graphical interface of the application? For Leonardi (2010), the latter seems to suffice, as he points to how different features in the photo-editing software Adobe Photoshop are material if and once they make a difference in a user's ability to accomplish his/her goal. In identifying and discussing the materiality of music streaming software, we may hence find a surface-level analysis sufficient.

A brief description of the system design may nevertheless be useful for identifying the matter of Spotify. The core function of Spotify is to enable end-users to play music with low latency and without stuttering (Kreitz and Niemelä, 2010). This user-experience goal points to the system design with clients (desktop and mobile Spotify applications) and the Spotify backend server sites. The backend sites cater for different related services, such as searching for and streaming music, managing playlists and connecting with peers. When users stream music, the data is obtained from the client local cache or the Spotify storage system in the backend site. The client backend architecture is crucial also for the social features of Spotify (Setty et al., 2013). Users can subscribe to or follow friends, playlists curated by others, or artists' pages. These subscription types next relate to different publication events. Friend feed notifies followers about music played, changes to playlists or new favourite artists, tracks or albums. The Spotify clients act as subscribers, and request subscription data through the access points to the Spotify backend service (Setty et al., 2013). 
This portrayal of Spotify as a system points to components that are invisible for end-users. These system components constrain and enable actions, and they are made material through visible features such as search, browse, play, create playlists, connect with peers, friend feeds, and features for sharing music. Spotify additionally includes editorial playlists. The 'Your Music' function, introduced in 2014, enable users to save albums and artists into their Spotify library.

These features are however different in terms of their reliance on users prompting certain actions. When a user plays music or saves an album, Spotify responds accordingly. Conversely, friend feeds appear to act on their own independently of human action; i.e. once I have connected to peers, the machine operates autonomously and automatically. There is consequently a need to distinguish between the materiality and the machine agency of streaming services.

\section{Machine agency}

Digital and networked systems increasingly act autonomously. These actions are often, if not always, initially designed by humans, yet it still makes sense to think of these systems as enacting agency. In Information Systems research, machine agency points to how machines play increasingly significant roles, influencing other human and machine actors and possible outcomes (Engen et al., 2016; Rose et al., 2005). Machines act autonomously, in ways that are inextricably linked with human agency; they 'facilitate and enable some parts of the human exercise of agency, but constrain other parts' (Rose et al., 2005: 15). I propose that machine agency must be an integral part of an affordance-approach to technology. If affordances relate to the action-potentials of an object, machine agency directs us towards perceiving certain action-potentials.

Although machines do not have intentions or motivations, their ways of enacting agency have consequences, and machine agency is relevant for understanding the way software is programmed to act on its own. Consider the role of algorithms for filtering and recommending content in Facebook (Bucher, 2012), or algorithmically recommended music, tuned to fit the listening patters and preferences of users (Morris, 2015: 452). Scholars typically warn against an account of algorithms as neutral (Beer, 2013; Crawford, 2016; Gillespie, 2013; Morris, 2015; Willson, 2017). Algorithms are not calculation machines that make autocratic decisions between variables; they function, are produced and modified in political spaces (Crawford, 2016: 79). They are the result of human agency in a market context and the intended and unintended ways codes work, 'seeking to frame meaning and curate experiences using a given set of raw materials (a database) and towards a distinct end (increasing listening time pointing users to other related commodities, etc.)' (Morris, 2015: 452). Algorithmic recommendations have taken in an increasingly central role in Spotify, for example with Spotify Discover Weekly launched in 2015; algorithmically generated playlists, personalized to take into account the taste profile for each user based on what they listen to and the playlists of Spotify-users with similar taste-profiles; filtered to account for 
both affinity and exploration.

Algorithmic recommendations depend on data, and users cannot escape providing data through their use of streaming services: part of the deal is to grant the service provider the opportunity to monitor, profile and analyse every single micro-action; combined into massive databases of tastes and preferences (Morris, 2015). Algorithms as step-by-step operations are written by humans always situated in an economic, cultural and political context. Yet it still remains relevant to ask whether and how music streaming services may potentially change how we relate to and listen to music. This question concerns the role technology plays in informing, if not determining, consumer behaviour.

\section{What does Spotify 'want'?}

The objective of the paper is to examine exploring and archiving of music. The affordances of interest are concomitantly formulated as 'explorable' and 'archivable'. Action-potentials for browsing music (material), connecting with friends (material), recommendation algorithms (machine agency), friend feeds (machine agency), and editorial playlists (material) point towards explorable as an affordance. In contrast, the opportunities to create playlists (material), save albums and artists in 'Your Music' (material), and play local music files stored on the computer (material) point towards archivable as an affordance. Spotify enables users to retrieve their recently played music, yet this list merely features the recent listening-history, and it is not a full, chronological account. This list of recently played music is hence transient, and is therefore not included as a machine agency feature pointing towards archivable.

If affordance concerns the action-potentials of an object, machine agency seems to interfere or push certain action-potentials over others. The machine agency of Spotify pushes people towards exploring and discovering music, whereas the personal and mnemonic meaning of music is supported via material features that ultimately rely on human action. Figure 1 responds to the first research question of how Spotify facilitates discovering, collecting and archiving music, yet is still incomplete without the required specifics of how humans act (the second research question). 


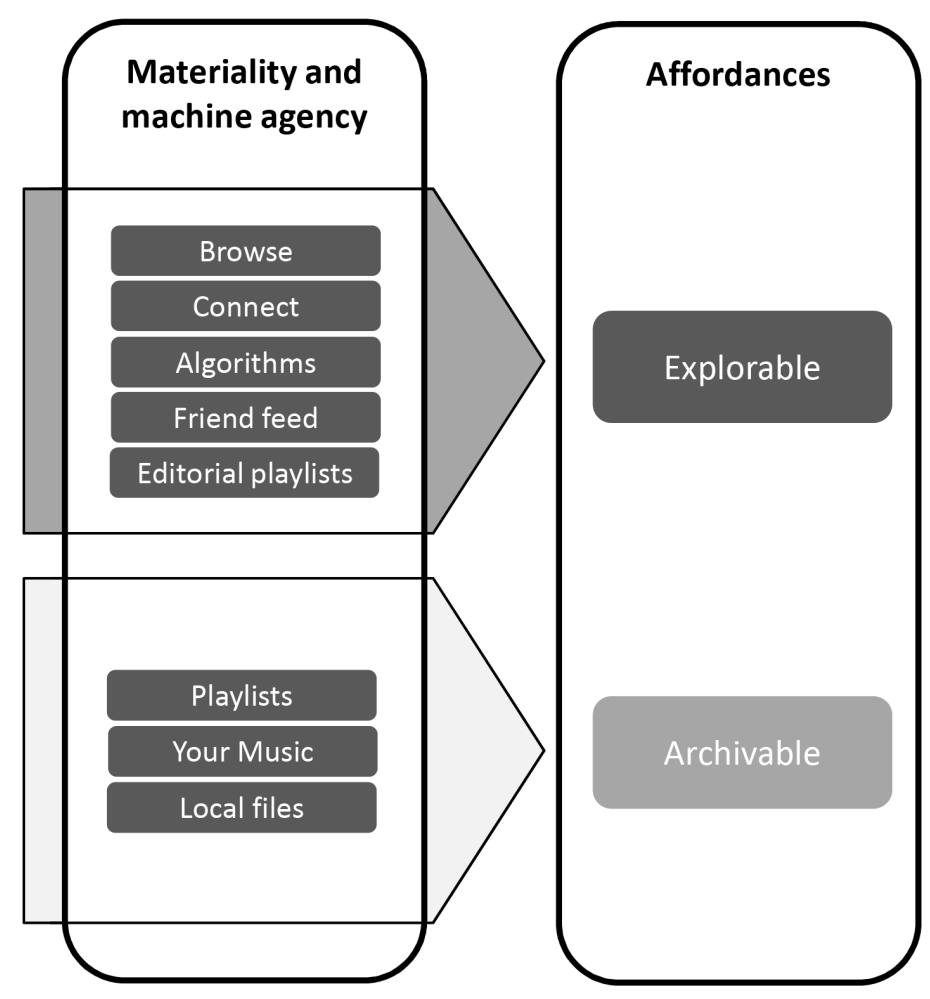

Figure 1: The materiality and machine agency of Spotify seem more strongly oriented towards explorable as an affordance than archivable.

\section{Method}

In order to examine how people express needs for exploring and archiving music, I rely on 23 focus group interviews with 107 users (aged 18-57, average age 28) of Spotify. These interviews were conducted between 2010 and 2013, and hence before Spotify introduced 'Your Music' and before the introduction of personalized recommendations such as 'Discover Weekly' and 'Your Daily Mix'. This study is also limited in terms of studying a relatively homogenous group; most participants being relatively young, urban and expressing an above-average interest in music.

Participants were recruited at the pop/rock festival Øya in Oslo, Norway. The interviews were scheduled after the festival and conducted face-to-face. In the interviews, participants were asked about their use of Spotify; what music means to them; and how they explore and discover music. The focus-group interviews were recorded, transcribed verbatim and then coded and analysed using Nvivo 11.

The analysis followed a two-step procedure. I first examined how each participant talked about exploring music and listening to music they are already familiar with, and grouped the participants into three categories based on whether they tend to use Spotify primarily for exploring music; for a combined pattern of exploring and listening to music they are already familiar with; or primarily for listening to music they are already familiar with. Next, I conducted a thematic analysis and generated codes and categories from the data. A thematic analysis allows for key themes to be determined prior to the analysis, yet the specific codes and categories emerge through close-reading and coding 
the data (Ezzy, 2002). Participants are anonymised in the below analysis, and referred to using fictive names.

\section{How people express needs for exploring and archiving music}

Table 1 presents an overview of the participants, grouped according to their listening patterns when using Spotify.

Table 1: Three types of streaming listening patterns among participants.

Listening pattern Example quotes

Explore:

31 participants

Average age: 30

15 women

16 men

Explore and

same old songs:

56 participants

Average age: 27

28 women

28 men
Christopher (30): 'It's cool to just come over something. To get lost in related artists, and to find new stuff, like related artists of related artists. I've found great music that I didn't know I was looking for'.

Elisabeth (27): 'You're being served with new music, and that's great (...). It opens up for new stuff that you weren't aware of'.

Anders (23): 'I listen a lot to music I already know. And I use related artists on Spotify. I like it, because I can check them out a bit. If I don't like it, I move on'.

Malin (25): 'I make playlists, I have different lists. I have one that I've entitled a specific year. And then when a new year comes, only the best ones make it to the new list. Because I kind of miss how it used to be, like that album used to be that holiday. I miss that, and I want it to be like that'.

Andreas (29): 'I don't listen to new music that much. I've

Same old songs: $\quad$ returned to what I used to listen to. I know of the music 13 participants before I play it'.

Average age: 26

6 women

7 men

Solveig (30): 'I feel like a fossil or dinosaur. I'm not very oriented towards checking out new music. I like the bands I've always liked'.

Participants who did not talk enough about listening patterns

Un-coded: 7 participants to be coded into any of the three categories.

Most participants value the opportunity to explore and discover music, yet they also remain attached to the music they are already familiar with. Table 1 hence hints at the importance for participants to have personal music collections, and hence archivable as 
an affordance. In the below analysis, I first discuss practices of exploring music, pointing towards explorable as an affordance, and next discuss practices towards playing the same old songs, pointing towards archivable as an affordance.

\section{Explore and discover}

A recurring theme among the participants is how easy access to vast music libraries make them inclined towards exploring and discovering music. The distinction between explore and discover is made in order to capture the practice of exploring the unknown with the aim of discovering new music that matters, where new denotes novel to the user and not (only) new releases. Participants talk about 'getting astray' or 'getting lost' in the 'the jungle of music'. Finding a path is compared to a journey where neither the route nor the destination is pre-given; or in the words of Morten (50), 'It's curiosity that drives me in this or that direction. And then I start listening to something I enjoy, that's what I like about Spotify: that I can click further, and start the big journey'. As a consequence of easy access to and availability of music, participants emphasize that they have become more open towards music they would not have listened to in a prestreaming era:

Erik (23): [Spotify] has increased the amount of time I listen to music. Old music too, or music that I'm not first sure I like. Like with Tom Waits, I've always been very ambivalent to him, just because of his voice. But now I like him a lot. That's because Spotify made it easier for me to listen to Waits without a lot of effort.

Magnus (26): I've been stuck in the music I discovered when I was about 18-19. Because I had it in my music-collection. I didn't have a way of finding music, besides through friends. I now listen to completely different genres. I listen to a lot more music, and I'm more open than I used to be.

Participants also explain their own volatile belonging to taste cultures as a consequence of growing older. They emphasize that music was a stronger identity-marker when they were younger, and that they no longer consider music to define who they are.

Ellen (27): I've been through my hippie-phase, my goth-phase, my electronicaphase, and I kind of lived each music-style in its entirety. It defined who I was. After having been through, like FIVE different phases, I started losing how music affiliates with my identity. And I started listening to absolutely everything.

The participants hence point to their acquired openness to music also as a consequence of growing older. Yet this life-phase effect does not disqualify how Spotify prompts users to explore music. In the next two sections I will address how participants explore music through peers, and how they explore music through algorithmic recommendations. 


\section{Exploring and discovering music through peers}

The opportunity to follow strong, weak and absent ties in Spotify is used as a way to explore and find music (Author): Specific strong ties are experienced as trustworthy providers of input to exploring music; and selected weak and absent ties, with whom participants experience a sense of musical fellowship, come across as credible and interesting musical peers. As Martin (26) reasons, 'You need a filter because there is so insanely much there. You need to start somewhere. And that makes it neat, or friends can recommend things.' Participants talk of shared playlists as providing easy access and input to music, particularly with regard to close friends and peripheral peers who are very interested and knowledgeable of music.

Heidi (26): You have the 'feed' if you've linked Spotify to Facebook. And you can see what your friends have listened to, and if several of your friends have listened to an artist or an album, and you see it in your [Spotify] feed; for me that's enough for thinking 'oh, I want to listen to that'. That way friends or acquaintances, regardless of whether they are close or distant friends, have some sort of power or status as opinion-leaders.

Friend feeds may also include music followers already have a relationship with. Yet when talking about the opportunities to follow others, participants emphasize the prospect of discovering new music. Relatedly, as I will discuss next, algorithmic recommendations are primarily emphasized as a way to explore and discover music.

\section{Algorithmic recommendations}

When participants talk about algorithmic recommendations they mainly point to Spotify's 'related artists' feature. These recommendations are determined for example by collaborative filtering algorithms, calculating similarities among users or items (Liu et al., 2014; Kjus, 2016). Such recommendations are valued as relevant, and several participants mention recommendations as partly explaining how they now belong to a wider variety of taste cultures:

Gunnar (32): I'm very conservative in terms of music-taste, like certain genres, hard and abysmal. But when I started streaming, the whole world opened. I've become this guy who also listens to calm and classical music. No longer only punk and hard-core, now it's all kinds of music. It's because of what Spotify recommended. It has changed me radically.

Elin (30): Spotify opened my horizon, or opened up to genres I wouldn't have bought in the record-store. And I like that thing up in the corner, if you're listening to an artist, it relates to TEN other artists. And you can browse them, and you keep on. Get across a lot of different artist that you wouldn't have listened to otherwise. 
The intentionality of the participants is evident: they rely on and use recommendations as a way to explore the abundance of music available: "I often use "related artists" to find music and to sit there and listen. Or to find new directions. I sometimes think of the selection, like who decided that? That this is related and this is not' (Helene, 31). Few participants reflect much on how algorithmic recommendations work, and Helene's comment about 'who decided that' does not reflect the general tendency. Their reliance on music streaming services and the way they use the recommendations are typically of a second-order control (Bandura, 2006), and participants rarely discuss how these systems operate. They do not express curiosity in the 'black box' matter of the streaming services; instead the outcomes of their own actions combined with what the machine does are interpreted as proofs these recommendations simply work.

The data-input to these algorithmic recommendations come from unknown peers: the music we play and how we sort music into playlists determine what is calculated as 'similar' for others. The algorithmic output, the recommendation, is as such always already an intermingling of machine agency and human action; although with regard to the latter, most likely unintentionally so (and hence not human agency): there is no escape from becoming part of the recommendation-system.

The analysis thus far suggests that the machine agency of Spotify plays a role in informing user-behaviour. Spotify invokes changes in the amount and genres of music participants listen to, opening up their taste-preferences. The participants overall express satisfaction with such nudging of their pre-Spotify taste-boundaries of music. Yet they also experience wariness with increasing impatience as a consequence of the urge to always continue exploring.

\section{Ephemeral music experiences}

A consequence of explorable as an affordance is listening-patterns becoming more ephemeral. Participants talk about losing focus, no longer being able to listen to fulllength albums, and always being on the move elsewhere.

Anne (29): I don't give the album attention anymore, because I jump too quickly between songs. It's kind of more ephemeral... I sometimes force myself to listen to a full album. But I get curious about where that track can take me, to things they recommend. I become 'DJSkippy, jumping between songs like crazy.

Camilla (38): I've become much more, like the 'google-generation's' relation to music: 'a little bit of this and that'. It used to be like 'yeah, I bought this album, and I listened to it a lot. (...) It used to be a lot more focused. It was deeper, but now I'm more like whimsical.

Listening to music through streaming services becomes an act of non-linear finding: digital storage, access technologies and search algorithms afford a tailored access to music, pushing the participants to always explore further. Remarks about being more 
impatient, always feeling the urge to find music that fits better with a mood or situation, and shame with not being able to focus on full-length albums were common in all focus-groups. Abundance, easy access and a drive towards exploring come with a cost: music becomes transient, with an added risk of music losing its attachment to a certain time and place:

Jonas (28): It used to be that you bought a CD and listened to it. Which made you listen to it in a certain time-setting. Now you just pick randomly, which makes it more difficult to associate an album with a certain occasion or epoch. But if I go back and choose songs from an album I used to listen to a lot, I do get taken back in time.

Nora (26): If I look back, there are a lot of songs that mean a lot for me in terms of linking with memories about something, or the lyrics relate to this or that. It's no longer like that. Now I just think 'oh, great song', or 'nice lyrics', but I don't think any more of it. I just throw it over in a playlist, and it plays in the background, you don't think about what you're listening to, you just know that it's somehow pleasant.

There is a sense of loss in these sentiments, as if the urge to explore needs to be countered by practices that ensure music can still be linked with an individualized history. The next part shows how the study participants take actions to create retrievable collections, their will to archive.

\section{Save and collect}

The argument in this part of the analysis is that Spotify, at least at the time of the datacollection, appeared inferior to physical collections or digital music collections in the form of local files. Finding and playing a song leaves too little trace for the user, and the music is at risk of being lost unless the user actively takes actions to retain it.

Emma (22): One thing that I miss, (...) or sometimes I feel I need to remember, like 'oh, I must remember THIS, I need to get back to this at some point'. So, there's no function that easily helps me find back to that artist. There are like a LOT OF artists passing by, that I really want to listen to, but then I forget.

Daniel (26): Are you talking about Spotify now? You can put a star on an album. Emma (22): Then they should perhaps be better at informing people about it.

Actions for saving music are not complex, yet these features are at risk of never becoming material for the user, as is the case for Emma. The lack of features for automated logging and sorting of music for users seems peculiar given the amount of data that music streaming services collect from users. Spotify collects and processes data on interactions with their service, including tracks played, but they have not fully remediated the music-collections people used to rely on: 
Anders (23): I'm kind of living in the old days. I love to buy CDs. I love to buy LPs. I want a big collection. But when I'm sitting down to listen to music [in Spotify], I go blank. I listen to the same band three times in a row. Because I can't remember all the bands that I want to listen to really. CDs are better that way, because I have them, I just need to play a CD. Spotify almost limits my choice.

Similar statements and nostalgia with the material collections of the past is a common theme in the interviews. The nostalgia with the LP and CD in this context is not about artwork or leafing through a pamphlet, but about physical albums as reminders of music that matters.

Magnus (26): I miss having my own overview of artists and albums. Like, 'THIS is my music; this is the fundament for my music'. If I create a playlist on iPhone [iTunes], I browse through all of my CDs and listen to some strange songs because often you listen to the best songs until they kind of become broken, whereas other songs grow on you with time. This is much more difficult with Spotify, and I miss it.

Spotify does not provide users with a comprehensive overview of music they have played. Logging music is possible by integrating Spotify with the third-party service last.fm, and this is a valued feature among the participants who in the interviews say they have done so. Yet the necessity of using a third-party service means most participants create playlists as a means to save music that matters.

\section{Curate and remember: Playlists as digital archives}

Creating, curating and managing playlists in music streaming services enable users to develop a sense of ownership to music (Hagen, 2015). Such patterns are typical also for the participants in the current study, yet the primary interest here is how most playlistpractices relate to a need to retain music.

Ann (22): I create playlists that I never delete. Usually either artist-playlists or singles. Usually music that I listen to a lot for a month or two, and then I'm done with those tunes. But it's nice to return to those playlists. They are my digital archive, and I rarely delete them.

As digital archives of personally curated music, playlists are emphasized as a bridge to the past in double sense. Music sorted into playlists may spark off memories of past experiences, and the playlist-feature appears as the closest analogy to music collections of the past: 'They are my digital archive', as explained by Ann. Playlists save music from the evanescent character of streamed music and provide a way of retrieving music. Jonas (28) maintains different types of playlists, including 'playlists for songs I don't want to forget'. Such sentiments are typical, and often also link music with a certain 
phase in life, or in the words of Kristine (24), to 'when I was 14, that was the time I loved hip-hop'. Julie (40) likewise says, 'The setting is important; suddenly you can be taken back in time or to a context'. Hence participants link music to personal meanings and life narratives, in similar ways as previous research has found for pre-streaming music-listening practices (Giles et al., 2007; Kibby, 2009; Turino, 1999).

Such accounts in particular relate to Turino's reasoning of how some music become indices that carry personal meanings: once their indexical relations to a certain time or place have become established, 'the index may still call to mind objects previously experientially attached' (Turino, 1999: 235). Certainly, some music is more or less devoid of affective and personal meaning beyond being ephemerally pleasurable. Yet music streaming services in their current form, where abundance and access call for active individuals who curate and maintain music in order to mitigate the risk of 'losing' music, require considering the long-term implications also in terms of the personal meaning of music. Music and music collections have always been a site of negotiating social status (Hesmondhalgh, 2008). Music streaming services provide access to vast libraries of music, but such access does not level the field, and there are likely distinct inequalities in people's interest and ability to curate 'their music'. The unsettled question is whether and how the materiality and machine agency of music streaming services should or could be better tuned to the personal and affective meanings of music.

\section{The art of playlisting: the soundtrack of our lives}

Playlists represent a value-added feature, yet one that is highly reliant on human action. The most devoted and skilful music listeners, who invest time and effort in curating music into different types of playlists, receive ample benefits in return: compilations tuned to fit different moods and everyday situations; always accessible, and always subject to further modifications:

Marius (28): I put together different lists from different artists, but it's always very specific in terms of what types of songs I want, and I always need to jump between playlists, different artists, and exactly those and those bands that I want to listen to next. What fits after this, dynamically, rhythmically or related to energy; if I'm tired or wired up. I can never just press play and let it go on. I always need to think of what I want as the next song.

Lars (22): Playlists based on a certain mood; I have several work-playlists that I have in the background while working. I don't have to think, it just runs in the background. When I make a new playlist, I play through it. And during the next two or three plays I change it considerably. If for example the transition from one track to another doesn't work very well. And then I let it be, and I can play it without annoying issues. 
Playlist-collections are compilations of music that matters and that are suited as soundtracks at work, at home, at the bus, doing everyday chores, while falling to sleep, working out, or while hanging out with friends. Playlists as soundtracks hence include both the curation of music that participants relish for the sonic and aesthetic quality, as well as music as experiential and functional add-ons, included for the appropriate beat and catchiness. There is a certain virtue in the act of saving and managing music. Prestreaming, buying an album or a digital file was sufficient for archiving it. In the era of streaming, finding and streaming a track leaves no permanent trace unless the listener takes further action.

\section{Concluding discussion}

So, what does the Spotify-machine 'want' and what do humans want when it comes to exploring music and archiving music? Figure 2 depicts explorable and archivable as affordances relative to the materiality and machine agency features of Spotify and human practice. Whereas participants value the opportunities to explore and discover music, their practices are equally directed towards archiving music.

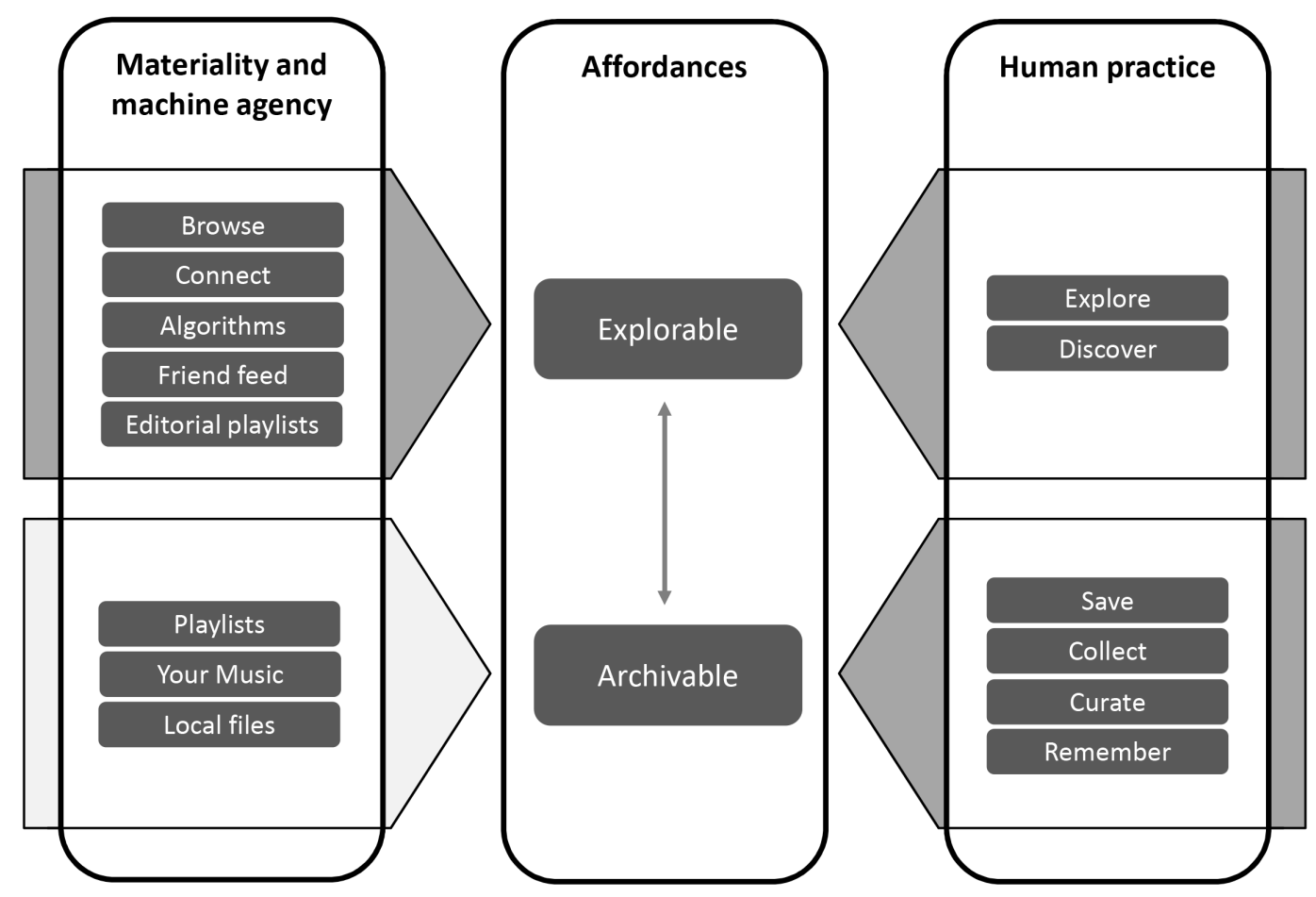

Figure 2: Explorable and archivable as affordances of Spotify, analysed as imbrications of materiality, machine agency and human practice.

The bidirectional arrow between explorable and archivable in Figure 2 refers to how the two are not trade-offs: what users archive relate to what they have explored, and what they explore relates to what they archive. The latter is even more the case after the introduction of personalized recommendations, where Spotify's recommendations are based on individual taste profiles: your playlists and the music you play serve as input 
to the recommendations you receive.

The central claim of this article nevertheless remains that Spotify appears skewed towards prompting users to explore rather than archive music. This claim can be countered as Spotify grants people opportunities to save music and create playlists. Yet it remains a legitimate claim, particularly if considering how the participants talk about music becoming ephemeral or transient. Add to this the seemingly stronger emphasis on machine agency for exploring. Whereas saving music and managing playlists depend on human action, Spotify acts as an autonomous agent with regard to tailoring and prompting recommendations towards users.

An important theoretical contribution of this article is delineating how objects with machine agency are different from material objects in terms of affordances. The action potentials of material objects can be considered as symmetrically constituted by what the objects provide relative to an active being. The action potentials of objects with machine agency interfere with this symmetry: the machine is designed and programmed to act on behalf of the human being, making certain affordances more perceivable than others. An affordance cannot be reduced to an object's features or reduced to how people choose to use an object. Still, when objects are programmed to act autonomously, we may raise the question of whether this comes with the cost of reduced agency to humans.

Much like affordances cannot be reduced to what people choose to do with objects, the personal meaning of music cannot be reduced to what people choose do with music (cf. Hesmondhalgh, 2008). Both types of enquiries need to address how technologies and cultural services and products are embedded in economic and political contexts. This has implications for the analysis of musical consumption and for the analysis of the business logic of music streaming services.

First, with regard to musical consumption, access to vast libraries of music per se is no social equalizer. The accounts of the participants suggest that they manage and curate music and hence make their own archives to cope with the volume and speed of cultural products (cf. Featherstone, 2000: 162-163). Yet the participants' ways of managing music reflect how they consider music to be important in their everyday lives. Hence, they likely express a stronger will to archive compared to people for whom music plays a less significant role. This will is coupled with an interest and ability to invest the required time and effort to curate and archive. This study only hints at how the will and ability to archive (and in a sense resist the push to explore) varies. Studies that build on empirical data from more heterogenous participants are needed to address questions of the limits of people's 'will to archive'.

Second, with Spotify as a case, it becomes central to discuss how the business logic of the service is reflected in the design of the service. If Spotify pushes certain behavioural patterns, can these patterns be explained partly as consequences of the interests of the different stakeholders involved in delivering the service? This question means moving from how to why Spotify is skewed towards exploring. Spotify argues that it is a strength that people increasingly use Spotify to discover music and that users 
listen to a greater variety of artists, and moreover points to their programmed features (e.g. Discover Weekly and Daily Mix) as 'driving this growth in artist diversity' (Erlandsson and Perez, 2017: un-paginated). Yet the why-question begs investigating for whom such user-patterns is more desirable. Which stakeholders benefit more when people explore more?

With regard to information providers and search engines, Gillespie (2017: 64) argues that 'those interested in having their information selected as relevant will tend to orient themselves toward these algorithmic systems, to make themselves algorithmically recognizable'. This paper asserts that Spotify appears to encourage (or push) users to explore, and does not delve into a discussion of the power to define what users are encouraged to explore, or whether and how big and small music providers adjust to be picked up as relevant. Future research needs to examine the logic of streaming, and how different players respond to this logic. Such efforts may benefit from steering clear of the black box metaphor and instead follow Bucher's (2018: 55) lead and ask 'how and under what circumstances different aspects of algorithms and the algorithmic are made available - or unavailable - to specific actors in particular settings.' Reverse engineering, experimenting with data inputs and data output, can give insight into how systems work (Bucher, 2018; Kitchin, 2017). Additionally, studies of how music industry actors (such as composers, musicians, labels, producers, publishers) relate to and adapt to music streaming services are needed to provide insight into the economics and politics of the production and distribution logics of streaming.

Networked systems are designed by humans situated in an economic, cultural and political context. My contention is that we can only properly examine the humanmachine networks we are now an entangled part of by granting interest in both the human and the machine part of these equations. This is even more important as the technologies we use are increasingly embedded with autonomous components, designed to act on their own, and in the case of Spotify appear to direct towards one behaviour over another; explore over archive. The notion of affordance is pertinent for such analyses, yet requires investigating what happens in the space between materiality and machine agency on the one hand and human practice on the other.

Acknowledgements not included for peer-review

\section{References}

Author

Beer D (2013) Popular culture and new media: the politics of circulation. Basingstoke: Palgrave Macmillan.

Bucher T (2012) Want to be on the top? Algorithmic power and the threat of invisibility on Facebook. New Media \& Society 14(7): 1164-1180.

Bucher T (2018) If... Then: Algorithmic Power and Politics. Oxford University Press. 
Crawford K (2016) Can an Algorithm be Agonistic? Ten Scenes from Life in Calculated Publics. Science, Technology \& Human Values 41(1): 77-92. DeNora T (2000) Music in Everyday Life. Cambridge: Cambridge University Press. Engen V, Pickering JB and Walland P. (2016) Machine Agency in Human-Machine Networks; Impacts and Trust Implications. In: Kurosu M (ed) Human-Computer Interaction. Novel User Experiences: 18th International Conference, HCI International 2016, Toronto, ON, Canada, July 17-22, 2016. Proceedings, Part

III. Cham: Springer International Publishing, 96-106

Erlandsson D and Perez J (2017) Listening Diversity Increases Nearly 40 Percent on Spotify. In: Spotify Insights. Retrieved from https://insights.spotify.com/br/2017/11/02/listening-diversity-spotify Ezzy D (2002) Qualitative Analysis: Practice and Innovation, London: Routledge. Featherstone M (2000) Archiving cultures. The British Journal of Sociology 51(1): 161184.

Featherstone M (2006) Archive. Theory, Culture \& Society 23(2-3): 591-596.

Gibson JJ (1979) The ecological approach to visual perception, Boston: Houghton Mifflin.

Giles DC, Pietrzykowski S and Clark KE (2007) The psychological meaning of personal record collections and the impact of changing technological forms. Journal of Economic Psychology 28(4): 429-443.

Gillespie T (2013) The Relevance of Algorithms. In: Gillespie T, Boczkowski P and Foot, K (eds) Media Technologies. Cambridge, MA: The MIT Press, 167-193. Gillespie T (2017) Algorithmically recognizable: Santorum's Google problem, and Google's Santorum problem. Information, Communication \& Society 20(1): 6380.

Hagen AN (2015) The Playlist Experience: Personal Playlists in Music Streaming Services. Popular Music and Society 38(5): 625-645.

Hesmondhalgh D (2008) Towards a critical understanding of music, emotion and selfidentity. Consumption, markets and culture 11(4): 329-343.

Hutchby I (2001) Conversation and Technology: From the Telephone to the Internet. Cambridge: Polity Press.

Kibby M (2009) COLLECT YOURSELF. Information, Communication \& Society 12(3): 428-443.

Kitchin R (2017) Thinking critically about and researching algorithms. Information, Communication \& Society, 20(1), 14-29.

Kjus Y (2016) Musical exploration via streaming services: The Norwegian experience. Popular Communication 14(3): 127-136.

Kreitz G and Niemelä, F (2010) Spotify - Large Scale, Low Latency, P2P Music-onDemand Streaming. Paper presented at the 10th international conference on peer-to-peer computing, Los Alamitos.

Latour B (2005) Reassembling the social, Oxford: Oxford University Press. 
Law J (1992) Notes on the theory of the actor-network: Ordering, strategy, and heterogeneity. Systems practice 5(4): 379-393.

Leonardi PM (2010) Digital materiality? How artifacts without matter, matter. First Monday 15(6).

Leonardi PM (2011) When flexible routines meet flexible technologies: Affordance, constraint, and the imbrication of human and material agencies. MIS Quarterly 35(1): 147-167.

Liu H, Hu Z, Mian A, Tian H. and Zhu X (2014) A new user similarity model to improve the accuracy of collaborative filtering. Knowledge-Based Systems 56: 156-166.

Marshall L (2014) W(h)ither Now? Music Collecting in the Age of the Cloud. In Marshall L, Laing D and Frith S (eds) Popular music matters: essays in honour of Simon Frith. Farnham: Ashgate, 61-72

Morris JW (2015) Curation by code: Infomediaries and the data mining of taste. European Journal of Cultural Studies 18(4-5): 446-463.

Nagy P and Neff G (2015) Imagined affordance: Reconstructing a keyword for communication theory. Social Media + Society 1(2).

Rose J, Jones M and Truex D (2005) Socio-theoretic accounts of IS: The problem of agency. Scandinavian Journal of Information Systems 17(1): 133-152.

Schrock AR (2015) Communicative affordances of mobile media: Portability, availability, locatability, and multimediality. International Journal of Communication 9(18).

Setty V, Kreitz G, Vitenberg R, van Steen M, Urdaneta G and Gimåker S. (2013) The hidden pub/sub of Spotify. Paper presented at the 7th ACM international conference on Distributed event-based systems, Arlington, Texas, USA.

Turino T (1999) Signs of Imagination, Identity, and Experience: A Peircian Semiotic Theory for Music. Ethnomusicology 43(2): 221-255.

van der Hoeven A (2018) Narratives of popular music heritage and cultural identity: The affordances and constraints of popular music memories. European Journal of Cultural Studies 21(2): 207-222

van Dijck J (2006) Record and Hold: Popular Music between Personal and Collective Memory. Critical Studies in Media Communication 23(5): 357-374.

Willson M (2017) Algorithms (and the) everyday. Information, Communication \& Society 20(1): 137-150 